\title{
Estimation of Effective Doses of Radiation for Patients with Novel Coronavirus Disease Undergoing Chest CT Scans
}

\author{
Shaban Alramlawy ${ }^{(1)}$ and I. Maamoun ${ }^{(1,2)}$ \\ (1) Faculty of Medicine Department of Critical Care-Cairo University Hospitals, Cairo University, Egypt. \\ ${ }^{(2)}$ Medical Physics Department, Kundiawa Genera Hospital,Kundiawa, Papua New Guinea Kundiawa, Papua New Guinea.
}

\begin{tabular}{l} 
ART ICLE INFO \\
\hline Article history: \\
Received: $13^{\text {th }}$ Apr. 2021 \\
Accepted: $6^{\text {th }}$ July 2021 \\
\hline
\end{tabular}

Keywords:

Effective dose,

COVID19,

Image quality,

CT chest.

\section{ABSTRACT}

Evaluating the hazards and beneficiaries of chest - CT imaging for patients of COVID-19 is considered of high concern because many patients had been imaged many times. There is a lack of information about the harm associated for patients of COVID-19. To estimate the effective doses for diagnosing Corona virus patients from chest CT scans, a total number of 160 suspected Covid-19 patients were classified into four groups (GP1(n=40), GP2(n=40) and GP3(n=40)).

All groups have undergone CT chest examination (Toshiba, Activion 16). The acquisition protocols for the different groups were as follows: GP $1((\mathrm{n}=40), 100 \mathrm{Kv}, 160 \pm 15 \mathrm{mAs}, \mathrm{GP2}$ $((n=40), 120 \mathrm{Kv}, 150 \pm 20 \mathrm{mAs}), \mathrm{GP3}((\mathrm{n}=40)$, were $120 \mathrm{Kv}$ Sure Exposure (SE), GP4 (n=40) , $100 \mathrm{Kv}$, Sure Exposure (SE)) . and the other parameters are (pitch=1.5; rotation time $=0.75 \mathrm{sec}$; matrix size 512x512), All images were reconstructed by Filtered Back Projection.

The CTDI volume for the different groups were: (GP1: 7.8 $\pm 0.09 \mathrm{mGy}$; GP2: 26.4 $\pm 0.25 \mathrm{mGy}$; GP3: $16.8 \pm 4.7 \mathrm{mGy} ;$ GP4: $5.5 \pm 0.1 \mathrm{mGy}, P<0.05)$. The values for DLP in the different groups were (GP1: $322.4 \pm 24.5 \mathrm{mGy}$. cm; GP2: $907.4 \pm 15 \mathrm{mGy} . \mathrm{cm}$; GP3: 512 $\pm 20 \mathrm{mGy} . \mathrm{cm}$; GP4: $250 \pm 10 \mathrm{mGy} . \mathrm{cm}, \mathbf{P}<\mathbf{0 . 0 5})$. The effective dose for the different groups were (GP1: 4.83 $\pm 0.36 \mathrm{mSv}$; GP2: 13.6 $\pm 0.225 \mathrm{mSv}$; GP3:7.68 $\pm 0.3 \mathrm{mSv}$; GP4: 3.7 $\pm 0.3 \mathrm{mSv}, \mathbf{P}<0.05$ ).

Contrast, CNR and SNR were insignificantly different $(\mathbf{P}>0.05)$ among all groups. GP1and GP4 have a highly significant noise $(\mathbf{P}<\mathbf{0 . 0 5})$, in comparison with GP2 and GP3.

In conclusion, this study demonstrates that chest $\mathrm{CT}$ performed with $100 \mathrm{kV}$ protocol and dose modulation (GP4) results in a significant reduction in the radiation dose used with other groups. Based on the obtained results, the proposed CT chest protocol Gp4 (100 Kv with mA modulation) was found to be reliable in the detection of COVID-19.

\section{INTRODUCTION}

Coronaviruses are a family of viruses which cause illness, such as respiratory diseases or gastrointestinal diseases. Respiratory disorders, such as Middle East Respiratory Syndrome (MERS-CoV) and Extreme Acute Respiratory Syndrome (SARS-CoV), can range from common colds to more serious illnesses. [1] The novel coronavirus $(\mathrm{CoV})$ is a new strain that has not been previously described in humans.

Once researchers decided exactly what the coronavirus was, they assigned a name to it (as in the case of COVID-19, the virus that causes it is SARSCoV-2).
There are broad uses of radiation in medicine. Nevertheless, considering the benefits of medical radiation exposure, harmful long-term health implications are a cause for concern.

Imaging can be useful in identifying patients with COVID-19, which is particularly useful in areas where there is a good access to imaging technology but limited access to reliable and fast laboratory testing. [2]

Chest computed tomography (CT) is known to be the primary diagnostic modality for evaluating patients with COVID-19 [3-5]. Many studies [6-13] have studied CT image manifestations in COVID-19 cases.

The most prevalent chest $\mathrm{CT}$ imaging features of COVID-19 pneumonia are peripheral ground-glass 
opacities (GGOs) and consolidation in the lower and middle lung regions, typically bilaterally distributed and with multi-lobar participation. [14-16]

Because of the importance of CT in the diagnosis and monitoring of patients with covid-19, they are at least exposed three times a month to ionizing radiation.

The aim of the present work was to estimate the effective doses for patients with Corona virus from chest CT scanning.

\section{MATERIAL AND METHODS (Patient population)}

This study was approved by the local ethical research committee, and a written informed consent was obtained from relatives of patients where there was no chance that a patient could be identified (or could identify themselves). Written informed consents were obtained. A total number of 160 suspected Covid-19 patients (age range, 19-87 years, mean age $58.8 \pm 23$ years) weighing mean $70 \pm 25 \mathrm{~kg}$ were enrolled in the study. All patients undergoing the procedure were subdivided into four groups. The mean number of days before hospital admission was $9.0 \pm 5.5$. At the time of hospital admission, the nasopharyngeal swab identified 106 patients $(63,8 \%)$ who had positive PCR results for COVID-and all the tested positive again in the following 3-5 weeks and their antero-posterior chest radiographs showed patchy ground-glass opacities. The chest CT scans showing diffuse ground-glass opacities with a certain consolidation region were also performed three times for all patients. The seriousness of the disease was mild to moderate, in general. The mean hospital stays, including critical care and intermediate care, was $33 \pm 6.9$ days. Table (1) illustrates different patients characteristics and $\mathrm{CT}$ acquisition parameters for all participating groups.

\section{Radiation dose calculations:}

The patient dose indices were CTDIvol, Dose Length Product (DLP) and effective dose. CTDIvol and DLP were collected from the summary page of patient file [16]. Subsequently, the effective dose (E) was calculated using $0.015 \mathrm{mSv} / \mathrm{mGy} . \mathrm{cm}$ as an average conversion factor $\left(\mathrm{E}_{\mathrm{DLP}}\right)$ for chest, abdomen and pelvis according to equation (1) [14]:

$$
\mathbf{E}=\operatorname{DLP} \times \mathbf{f}
$$

Where $\mathrm{f}$, measured in units of $\mathrm{mSv} /(\mathrm{mGy} . \mathrm{cm})$, is a body region-specific conversion factor.

\section{Image Quality Assessment:}

The measured parameters of image quality were Contrast, Noise $(\sigma)$, Signal to noise Ratio (SNR) and Contrast to noise Ratio (CNR).

Image quality indices were measured by drawing Region of Interests (ROIs). The location and size of ROIs were consistent, as shown in Figure 1 [17]. The sizes of ROIs were measured at ROI (1): $240 \pm 10 \mathrm{~mm}^{2}$ and ROI (2): $255 \pm 10 \mathrm{~mm}^{2}$ for GGO (figure 1) and background equations (2, 3 and 4$)$ respectively.

$$
\text { Contrast =ROI (HU) }- \text { BKG (HU) / ROI(HU) + BKG(HU) }
$$

$$
\text { Noise }=\sigma / N=1 / \sqrt{ } N
$$

Where $N$ is the number of counts per unit area in the image

$$
\mathbf{S N R}=\frac{R O I(H U)}{\sigma}
$$

Each ROI was calculated for the CT value and the standard deviation (SD). Contrast to noise ratio (CNR) is defined as the ratio of the difference of signal intensities of two regions of interest to the background noise as shown in equation (5) [17]:

$$
\begin{aligned}
& \mathrm{CNR}=\text { Contrast } / \text { Noise } \\
& \mathrm{CNR}=\frac{\left|M_{o}-M_{b}\right|}{\sigma_{b}}
\end{aligned}
$$

\begin{tabular}{|c|c|c|c|c|c|c|c|c|}
\hline \multirow{2}{*}{ No. of group } & \multirow{2}{*}{ No. of patients } & \multirow{2}{*}{ Mean of age } & \multirow{2}{*}{ Mean of weight } & \multicolumn{5}{|c|}{ Acquisition protocol } \\
\hline & & & & kv & mAs & pitch & R.T sec & M.S \\
\hline Group 1 & 40 patients & $54.6 \pm 26 y$ & $73 \pm 25 \mathrm{~kg}$ & 100 & $160 \pm 15$ & 1.5 & 0.75 & $512 \times 512$ \\
\hline Group 2 & 40 patients & $56.8 \pm 17.7 \mathrm{y}$ & $74 \pm 22 \mathrm{~kg}$ & 120 & $150 \pm 20$ & 1.5 & 0.75 & $512 \times 512$ \\
\hline Group 3 & 40 patients & $52.9 \pm 25 y$ & $70 \pm 27 \mathrm{~kg}$ & 120 & Sure Exposure & 1.5 & 0.75 & $512 \times 512$ \\
\hline Group 4 & 40 patients & $57.5 \pm 23.8 \mathrm{y}$ & $75 \pm 27 \mathrm{~kg}$ & 100 & Sure Exposure & 1.5 & 0.75 & $512 \times 512$ \\
\hline
\end{tabular}

Where $M_{o}$ is the average Hounsfield unit of ROI at organs (GGO), $\mathrm{Mb}$ is the average Hounsfield unit of ROI at background (Lung) and $\sigma_{b}$ is the background standard deviation.

Table (1): The patients characteristics and CT acquisition parameters for all groups 


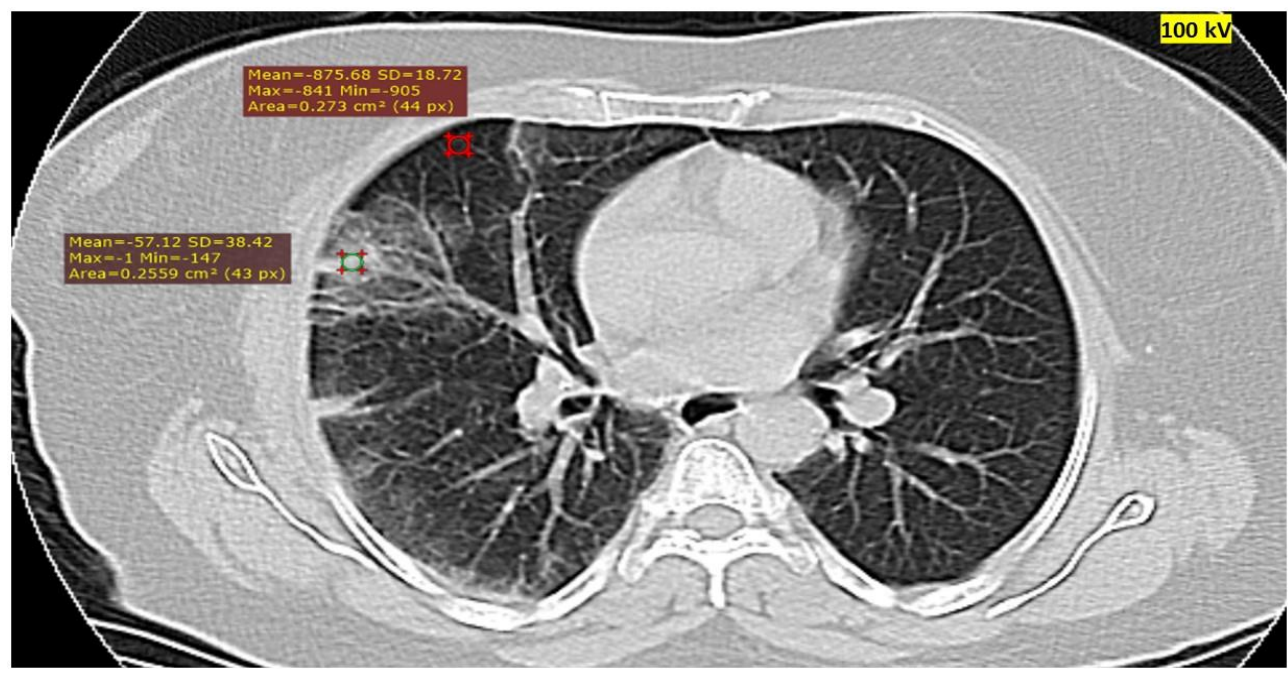

Fig. (1): Computer tomography showing the dropping of 2 regions of interest at Ground Glass Opacities (GGO) and Background

\section{Subjective assessment:}

Four radiologists (R1, R2, R3, and R4) used a scale of $0-3$ to measure the degree of image quality. Score 0 reflected inaccurate diagnostic image. Score 1 was demonstrative of a mild effect that affected the diagnosis. Score 2 suggested a weak noise that however, it was detected. Score 3 indicated that no items exerted an impact on the accuracy of the diagnosis.

\section{Statistical analysis:}

SPSS 19.0 software (IBM, Chicago, Ill) was used for statistical analyses. All experimental results were expressed by mean $\pm \mathrm{s}$. Differences were considered statistically significant at a $\mathrm{p}$ value $<0.05$.

\section{RESULTS}

The dose calculation parameters were highly significant $(\mathrm{P}<0.05)$ in GP2 in comparison with GP1, GP3 GP4 (Table 2 and figure 2).

GP4 had the lowest significant $(\mathrm{P}<0.05)$ effective dose in comparison with all groups (Table 2 and Figure2).

Table (2): Radiation dose measurements for different groups GP1, GP2, GP3 and GP4 per one CT chest scan

\begin{tabular}{cccccc}
\hline Parameter & GP1 & GP2 & GP3 & GP4 & P Value \\
\hline $\begin{array}{c}\text { CTDIvol } \\
(\mathbf{m G y})\end{array}$ & $7.8 \pm 0.09$ & $26.4 \pm 0.25$ & $16.8 \pm 4.7$ & $5.5 \pm 0.1$ & $<0.05$ \\
$\begin{array}{c}\text { DLP }(\mathbf{m G y} \times \\
\mathbf{c m})\end{array}$ & $322.4 \pm 24.5$ & $907.4 \pm 15$ & $512 \pm 20$ & $250 \pm 10$ & $<0.05$ \\
$\begin{array}{c}\text { Effective dose } \\
(\mathbf{m S v})\end{array}$ & $4.83 \pm 0.36$ & $13.6 \pm 0.225$ & $7.68 \pm 0.3$ & $3.7 \pm 0.3$ & $<0.05$ \\
\hline
\end{tabular}

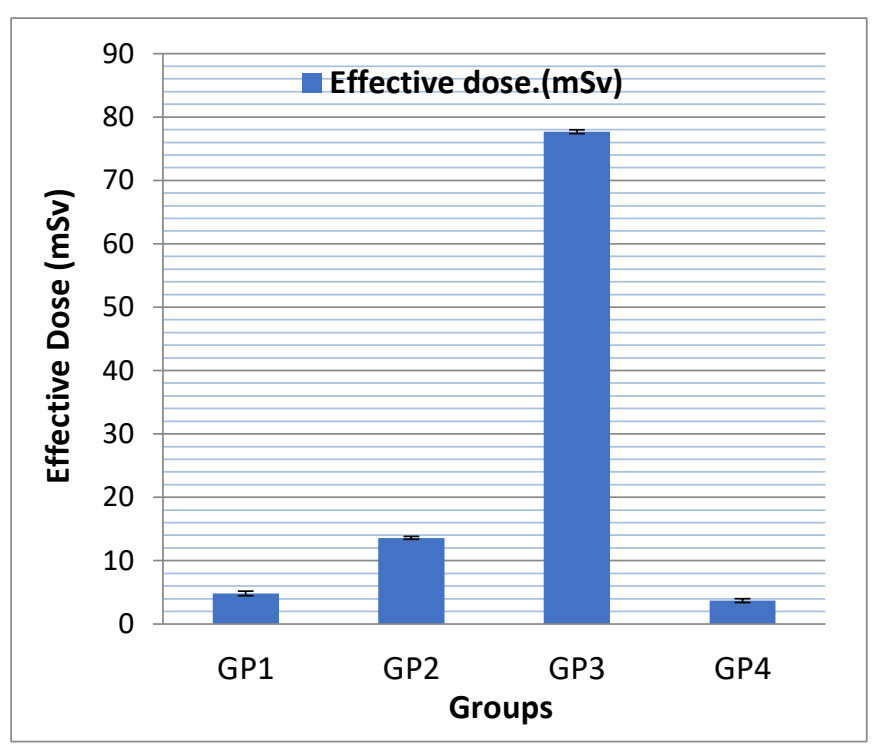

Fig. (2): The effective dose for one $\mathrm{CT}$ chest scan in $\mathrm{mSv} \pm \mathrm{SD}$ for different groups GP1, GP2, GP3 and GP4 sure exposure, where $\mathbf{P}<0.05$

Radiologists R1, R2, R3 and R4 graded all images of all groups by 3 (no effect on the accuracy of diagnosis), where no significant difference $\mathrm{P}>0.05$.

Patient data analysis showed an insignificant difference $(\mathrm{P}>0.05)$ of as regards Contrast, CNR and SNR in all groups, as illustrated in Table 3 and Figures 3,4 . However, the noise showed a highly significant difference $(\mathrm{P}<0.05)$ for GP 1, GP2 and GP4 in comparison with GP3. 
Table (3): Image quality parameters for all groups expressed in Mean \pm SD

\begin{tabular}{|c|c|c|c|c|c|c|c|c|}
\hline Groups & Contrast & $\begin{array}{c}\text { Contrast } \\
\text { SD }\end{array}$ & noise & $\begin{array}{c}\text { noise } \\
\text { SD }\end{array}$ & SNR & $\begin{array}{c}\text { SNR } \\
\text { SD }\end{array}$ & CNR & CNR SD \\
\hline GP1 & 0.47 & 0.23 & 53.3 & 17.2 & 11.7 & 6.4 & 19.029 & 9.4 \\
\hline GP2 & 0.5 & 0.27 & 36.2 & 18.8 & 31.3 & 27.7 & 38.102 & 10.4 \\
\hline GP3 & 0.43 & 0.18 & 27.1 & 12.2 & 27.5 & 15.3 & 32.034 & 12.1 \\
\hline GP4 & 0.39 & 0.22 & 50.2 & 15 & 10.5 & 6.0 & 18.3 & 14.5 \\
\hline
\end{tabular}

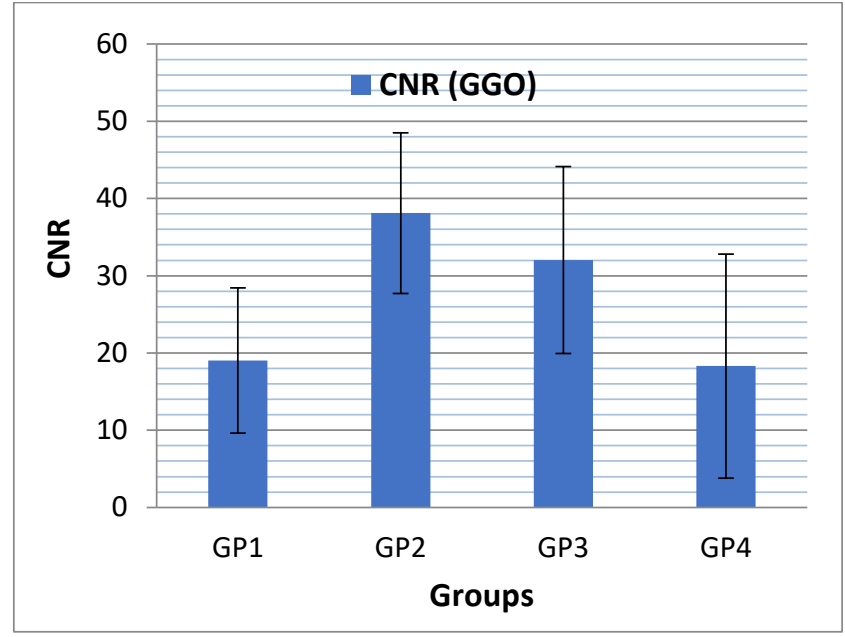

Fig. (3): The Contrast to noise Ratio (CNR) of Ground Glass Opacities (GGO) for different groups GP1, GP2, GP3 and GP4, where P>0.05

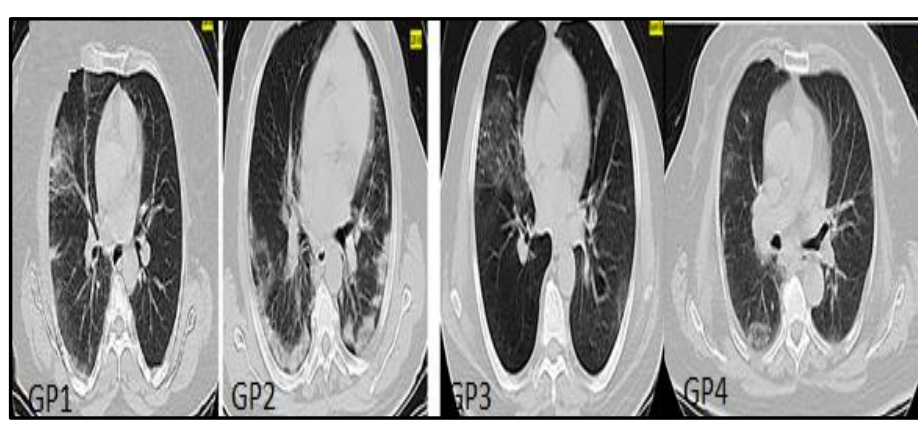

Fig. (4): computer tomography of axial cut is showing the image quality of Ground Glass Opacities (GGO) for different groups GP1, GP2, GP3 and GP4

The total effective dose of covid-19 patients for the three CT chest (Follow up) is shown in Table (4). As regards the capacity to detect COVID-19 pneumonia, there was no statistically significant difference between the different groups of CT images found. At the same time, the low dose protocol (GP4) in our analysis was associated with a reduction of approximately $48 \%$ in the mean effective dose value relative to $\mathrm{GP} 2(120 \mathrm{Kv})$.
Table (4): The total effective dose of three CT Chest (Follow up) for Covid-19 Patients for different groups GP1, GP2, GP3 and GP4

\begin{tabular}{|c|c|c|c|c|c|c|}
\hline Parameter & GP1 & GP2 & GP3 & GP4 & $\begin{array}{c}\text { The mean } \\
\text { hospital stay }\end{array}$ & $\begin{array}{c}\text { PCR for } \\
\text { COVID } \\
19\end{array}$ \\
\hline $\begin{array}{l}\text { Mean of Total } \\
\text { Effective Dose } \\
\text { per } 3 \text { CT } \\
\text { Chest scans }\end{array}$ & 13.13 & 38.8 & 20.1 & 10.2 & $33 \pm 6,9$ days & $3 \pm 1$ \\
\hline$P$ - value & 0.04 & 0.05 & 0.03 & 0.04 & 0.05 & 0.05 \\
\hline
\end{tabular}

\section{DISCUSSION}

With the high rate of infection resulting from the emerging corona virus (Covid-19), and the diversity of treatment and diagnosis protocols, the frequency of the use of a chest CT scan has increased in addition to assessing the diagnostic value of low dose protocol CT images. The current study shed light on the radiation exposure of Covid-19 patients undergoing CT Chest examination.

The cancer risk increases at a rate of $0.01 \% / \mathrm{mSv}$ exposure, so the hazard associated with radiation exposure of Covid-19 patients must be reduced during $\mathrm{CT}$ chest scan (reference required).

Reducing kv from 120 (GP2 and Gp3) to 100(GP1 and Gp4) in the current study caused a reduction in the intensity of X- ray photons produced by power 3 and the average energy. Subsequently all dose parameters were decreased by greater than $50 \%$ significantly $(\mathrm{P}<0.05)$ (Figure 2 and Table 2).

The diagnostic value should be maintained with $\mathrm{Kv}$ reduction as much as possible by $\mathrm{mA}$ modulation (SureExposure). The $\mathrm{mAs}$ modulation in $\mathrm{x}, \mathrm{y}$ and $\mathrm{z}$ directions causes a variation of intensity of $X$ Ray beam according to the patient density . The mAs modulation of $120 \mathrm{KV}$ (GP3) with patient size significantly reduced the patient dose while maintaining the same noise level compared to $120 \mathrm{KV}(\mathrm{GP} 2)$ Figure 2, Table 1).

These results agree with Leschka et al[18] who obtained a 25 percent reduction in the radiation dose while reducing tube voltage from $120 \mathrm{kV}$ to $100 \mathrm{kV}$ using the same tube current-time product ( $330 \mathrm{mAs}$ ). Also, the results are in line with those of Tobias Pflederer et al [19] who showed that their research achieved a nearly 39 percent reduction in radiation dose. Huda W et al[20] concluded that based on theoretical considerations, a dose reduction of approximately 40 percent could be predicted since the dose is related to the square voltage of the tube. 
Tube current-time product (mAs) is another aspect that affects the efficient dose (mSv). Although the relationship between the current-time product of the tube and the effective dose is linear, lowering the current-time product of the tube results in higher image noise and may therefore adversely affect the quality of the image.(21)

Four radiologists assessed all images and found no significant difference in image quality and the diagnostic value of GGO for all groups of COVID-19 patients.

The qualitative assessment was supported by the quantitative measurements of image quality indices that revealed reduction in the mean value of Contrast, noise, SNR and CNR with low $\mathrm{Kv}$ groups against high $\mathrm{Kv}$. Reducing Kv in GP1 and GP4 caused a reduction in the number of photons produced and transmitted through the patient to reach the detectors. The smaller number of photons (high noise) would not be enough to form the full image data for reconstruction. Using $\mathrm{mA}$ modulation maintains the noise level with different body contours that produces more homogenous images (as in GP4). However, the statistical analysis revealed that no significant difference in Contrast, SNR and $\mathrm{CNR}(\mathrm{P}>0.05)$, while a significant difference $(\mathrm{P}<0.05)$ in noise of GP3 was noted against all groups.

Tube current-time product (mAs) is another aspect that affects the efficient dose $(\mathrm{mSv})$. Although the relationship between the current-time product of the tube and the effective dose is linear, lowering the current-time product of the tube results in a higher image noise and may therefore adversely affect the quality of the image.(21).

Georgios Kareliotis[22] stated that although both tube potential and current-time product affect image quality and radiation dose, the former does it in a more drastic way. For low-contrast protocols, tube potential reduction down to the $90 \mathrm{kVp}$ region affects image quality, in contrast to transition from 140 to $120 \mathrm{kVp}$. For high-contrast protocols the low $\mathrm{kVp}-$ high $\mathrm{mAs}$ combination seems to be the best choice.

Gill MK[23] found that reducing the tube voltage from 120 to $100 \mathrm{kV}$ in CTPA allows a significant reduction in the radiation dose without significant loss of diagnostic image quality where the effective radiation dose for the 100 and $120 \mathrm{kV}$ scans was 3.2 and $6.8 \mathrm{mSv}$, respectively.

The repetition of CT chest for Covid-19 Patients enforces using low dose $\mathrm{CT}$ acquisition parameters that reduce the exposure to radiation. By using a tube voltage of $100 \mathrm{kV}$ instead of $120 \mathrm{kV}$ (the most suggested value), nearly $31 \%$ mean image quality ratings were well maintained, with no major variations in the quality of the image whether subjectively measured or in objective parameters such as the CNR and SNR. Therefore, when performing contrast-enhanced chest scan in non-obese patients, we actually support the use of lower tube voltages with dose modulation techniques.

Radiation exposure in a chest CT scan is influenced by several factors: First, tube voltage and the scanning length. Second, the speed of table movement (pitch) affects the duration of exposure and therefore radiation dose.

The diagnostic importance is maintained by the need for suitable methodologies to improve and decrease exposure of coronavirus patients to radiation.

A covid 19 patient usually undergoes between one and three CT scans and sometimes more than that. In this research paper our goal was to measure and determine the effective radiation doses for these tests, by using the following:

Four groups of patients were examined and the results were as follows:

In the Gp1 and Gp4 imaging technique, using a lowered tube voltage resulted in a significant decrease in contrast mean, SNR mean and CNR mean, and some markedly increased image noise compared to GP2 and GP3 image quality specifications, that hadenhanced contrast mean, SNR mean and CNR mean, and reduced significantlyimage noise quality parameters. Reducing $\mathrm{KV}$ from 120 to $100 \mathrm{kv}$ had a great effect on the reduction of the intensity of radiation and the average energy of the X Ray beam. Thus, a patient dose of 100 $\mathrm{Kv}$ (GP1 and GP4) was decreased by $50 \%$ in comparison with $120 \mathrm{Kv}$ (GP3 and GP4)) Figure 2 ,Table 4) .

These results agree with those of Leschka et al-[18] who obtained a 25 percent reduction in the radiation dose while reducing tube voltage from $120 \mathrm{kV}$ to $100 \mathrm{kV}$ using the same tube current-time product $(330 \mathrm{mAs})$ and are also in agreement with Tobias Pflederer et al [19] who showed that their research achieved a nearly 39 percent reduction in the radiation dose. Huda $\mathrm{W}$ et al. concluded that based on theoretical considerations, a dose reduction of approximately 40 percent could be predicted since the dose is related to the square voltage of the tube [20].

The mAs modulation in the $\mathrm{x}, \mathrm{y}$ and $\mathrm{z}$ directions causes a variation of intensity of $X$ Ray beam according to the patient's density. The mAs modulation of $120 \mathrm{KV}$ (GP3) with patient size significantly reduces the 
patient's dose while maintaining the same noise level compared to $120 \mathrm{KV}(\mathrm{GP} 2)$ Figure 2, Table 1). Tube current-time product (mAs) is another aspect that affects the efficient dose $(\mathrm{mSv})$. Although the relationship between the current-time product of the tube and the effective dose is linear, lowering the current-time product of the tube results in higher image noise and may therefore adversely affect the quality of the image.[21]

Georgios Kareliotis concluded that although both tube potential and current-time product affected the image quality and radiation dose, the former did it in a more drastic way. For low-contrast protocols, tube potential reduction down to the $90 \mathrm{kVp}$ region affects the image quality, in contrary to transition from 140 to 120 $\mathrm{kVp}$. For high-contrast protocols the low $\mathrm{kVp}-$ high $\mathrm{mAs}$ combination seems to be the best choice [22].

In the current study, four radiologists assessed all images and did not observe any significant difference in image quality and the diagnostic value of GGO for all groups of COVID-19 patients.

The reduction in CNR among all groups was not significant to assess the changes of lung in covid-19 patients (Figures 3 and 4).

The quantitative assessment of image quality revealed that the use of a lower tube voltage in the Gp1 and Gp4 scanning protocol resulted in a substantial decrease in contrast mean, SNR mean and CNR mean, as well as significantly higher image noise compared to GP2 and GP3 image quality parameters. The latter improved contrast mean, SNR mean and CNR mean, and significantly lowered image noise and this is illustrated in Table (3). The results of the present study agreed with those of Mannudeep K. Kalra who recommended against routine use of diagnostic imaging for the diagnosis of COVID-19 pneumonia unless there was a lack of availability to RT-PCR or immunoassays in patients with moderate to severe disease, worsening respiratory status, or a suspicion of cardiopulmonary complications. They recommended that a chest $\mathrm{CT}$ when indicated, should be performed with a low-dose, single-phase protocol using fast scanning techniques to minimize motion artifacts.[24]

\section{CONCLUSION}

The results of the present investigation reveals that CT chest protocol Gp4(100 Kv with mA modulation) was found to be reliable in the detection of COVID-19 pneumonia in daily practice with a substantial reduction in radiation dose modulation. It is also recommended that no chest CT scan should be conducted unless the
Corona virus analysis is positive, and that the procedure should not be repeated unless it is appropriate to prevent exposure to high doses of radiation.

\section{REFERENCES}

[1] World Health Organization. Coronavirus. Available from: https://www.who.int/health-topics/coronavirus (Accessed 14 March 2020)

[2] Centers for Disease Prevention and Control. Information for Health Departments on Reporting a Person Under Investigation (PUI), or Presumptive Positive and Laboratory-Confirmed Cases of COVID19 https://www.cdc.gov/coronavirus/2019ncov/php/reporting-pui.html Accessed 14 March 2020

[3] Zhou S, Wang Y, Zhu T, Xia L. CT Features of Coronavirus Disease 2019 (COVID-19) Pneumonia in 62 Patients in Wuhan, China. American Journal of Roentgenology. 2020 Jun;214(6):1287-1294.

[4] Ng M, Lee EY, Yang J, Yang F, Li X, Wang H, Lui MM, Lo CS, Leung B, Khong P, Hui CK, Yuen K, Kuo MD. Imaging Profile of the COVID-19 Infection: Radiologic Findings and Literature Review. Radiology: Cardiothoracic Imaging. 2020 Feb .

[5] Bernheim A, Mei X, Huang M, Yang Y, Fayad ZA, Zhang N, Diao K, Lin B, Zhu X, Li K, Li S, Shan H, Jacobi A, Chung M. Chest CT Findings in Coronavirus Disease-19 (COVID-19): Relationship to Duration of Infection. Radiology. 2020 Jun.

[6] Pan Y, Guan H, Zhou S, Wang Y, Li Q, Zhu T, Hu Q, $\mathrm{Xia}$ L. Initial CT findings and temporal changes in patients with the novel coronavirus pneumonia (2019nCoV): a study of 63 patients in Wuhan, China. Eur Radiol. 2020 Jun 13;30(6):3306-3309.

[7] Zhao W, Zhong Z, Xie X, Yu Q, Liu J. CT Scans of Patients with 2019 Novel Coronavirus (COVID-19) Pneumonia. Theranostics. 2020;10(10):4606-4613.

[8] Yoon S, Lee K, Kim J, Lee Y, Ko H, Kim K, Park CM, Kim YH. Chest Radiographic and CT Findings of the 2019 Novel Coronavirus Disease (COVID-19): Analysis of Nine Patients Treated in Korea. Korean J Radiol. 2020 Apr;21(4):494-500.

[9] Zhao W, Zhong Z, Xie X, Yu Q, Liu J. Relation Between Chest CT Findings and Clinical Conditions of Coronavirus Disease (COVID-19) Pneumonia: A Multicenter Study. AJR Am J Roentgenol. 2020 May;214(5):1072-1077.

[10] Zhou Z, Guo D, Li C, Fang Z, Chen L, Yang R, Li X, Zeng W. Coronavirus disease 2019: initial chest CT findings. Eur Radiol. 2020 Aug;30(8):4398-4406.

[11] Chung M, Bernheim A, Mei X, Zhang N, Huang M, Zeng X, Cui J, Xu w, Yang Y, Fayad ZA, Jacobi A, Li Arab J. Nucl. Sci. Appl., Vol. 54, 4, (2021) 
K, Li S, Shan H. CT Imaging Features of 2019 Novel Coronavirus (2019-nCoV) Radiology. 2020 Apr;295(1):202-207.

[12] Li X, Zeng W, Li X, Chen H, Shi L, Li X, Xiang H, Cao Y, Chen H, Liu C, Wang J. CT imaging changes of corona virus disease 2019 (COVID-19): a multicenter study in Southwest China. J Transl Med. 2020 Apr 06;18(1):154.

[13] Li Y, Xia L. Coronavirus Disease 2019 (COVID-19): Role of Chest CT in Diagnosis and Management. AJR Am J Roentgenol. 2020 Jun;214(6):1280-1286.

[14] National Council on Radiation Protection and Measurements. Ionizing radiation exposure of the population of the United States. Bethesda, MD: National Council on Radiation Protection and Measurements; 1987. Report No. 93.NCRP Report No. 93.

[15] Brenner DJ, Hall EJ. Computed tomography: anincreasing source of radiation exposure. N Engl JMed 2007; 357:2277-2284 .

[16] Yu L, Bruesewitz MR, Thomas KB, Fletcher JG, Kofler JM, McCollough CH. Optimal tube potential for radiation dose reduction in pediatric CT: principles, clinical implementations, and pitfalls. RadioGraphics 2011; 31:835-848

[17] Bushberg, Jerrold T. "The essential physics of medical imaging "/ Jerrold T. Bushberg. — 3rd ed. 2012 by LIPPINCOTT WILLIAMS \& WILKINS, a WOLTERS KLUWER

[18] Leschka S, Stolzmann P, Schmid FT, et al. Low kilovoltage cardiac dual-source CT: attenuation, noise, and radiation dose. Eur Radiol 2008; 18: 1809-1817

[19] Tobias Pflederer, Larissa Rudofsky , Dieter Ropers , Sven Bachmann - Image Quality in a Low Radiation Exposure Protocol for Retrospectively ECG-Gated Coronary CT Angiography .American Roentgen Ray Society. AJR 2009; 192:1045-1050, 0361

[20] Huda W, Scalzetti EM, Levin G. Technique factors and image quality as functions of patient weight at abdominal CT. Radiology 2000; 217: 430-435

[21] Shemesh J, Evron R, Koren-Morag N, et al. Coronary artery calcium measurement with multi-detector row $\mathrm{CT}$ and low radiation dose: comparison between 55 and 165 mAs. Radiology 2005.

[22] Georgios Kareliotis STUDY OF kVp AND mAs EFFECT ON RADIATION DOSE AND IMAGE QUALITY IN COMPUTED TOMOGRAPHY, National Technical University of Athens, Thesis April 2015, DOI: 10.13140/RG.2.2.32266.06083
[23] Gill MK, Vijayananthan A, Kumar G, Jayarani K, Ng $\mathrm{KH}$, Sun Z. Use of $100 \mathrm{kV}$ versus $120 \mathrm{kV}$ in computed tomography pulmonary angiography in the detection of pulmonary embolism: effect on radiation dose and image quality. Quant Imaging Med Surg 2015;5(4):524-533. doi: 10.3978/j.issn.22234292.2015.04.04

[24] Mannudeep K. Kalra \& Fatemeh Homayounieh \& Chiara Arru \& Ola Holmberg \& Jenia Vassileva Chest CT practice and protocols for COVID-19 from radiation dose management perspective Received: 17 April 2020 /Revised: 5 June 2020 /Accepted: 12 June 2020 \# European Society of Radiology 2020.

Table (5) of abbreviated words

\begin{tabular}{|c|c|}
\hline $\mathrm{CT}$ & computed tomography \\
\hline R0I & Region Of Interests \\
\hline SNR & Signal to noise Ratio \\
\hline FDG & fluorodeoxyglucose \\
\hline GGO & ground-glass opacity \\
\hline MERS & Middle East respiratory syndrome \\
\hline MERS-CoV & $\begin{array}{l}\text { Middle East respiratory syndrome } \\
\text { coronavirus }\end{array}$ \\
\hline $\mathrm{NCP}$ & novel coronavirus pneumonia \\
\hline $\mathrm{PET} / \mathrm{CT}$ & $\begin{array}{l}\text { positron emission } \\
\text { topography/computed tomography }\end{array}$ \\
\hline RT-PCR & $\begin{array}{l}\text { reverse transcription-polymerase } \\
\text { chain reaction }\end{array}$ \\
\hline SARS & severe acute respiratory syndrome \\
\hline SARS-CoV & $\begin{array}{l}\text { severe acute respiratory syndrome } \\
\text { coronavirus }\end{array}$ \\
\hline COVID-19 & Coronavirus disease 2019 \\
\hline CNR & Contrast to Noise Ratio \\
\hline $\mathrm{R}$ & Radiologists \\
\hline GP & Group \\
\hline CTDI & CT dose index \\
\hline $\mathrm{kV}$ & Kilovolt \\
\hline
\end{tabular}

